\title{
10 Nation und Nationalität
}

Dieses Kapitel wurde bei Erstveröffentlichung ohne die korrekte Creative Commons Lizenz veröffentlicht. Die korrekte Lizenz finden Sie am Ende des Kapitels.

Ein Erratum zu diesem Kapitel ist verfügbar unter DOI 10.1007/978-3-658-12533-2_14

(C) SINUS Markt- und Sozialforschung GmbH 2016

M. Calmbach et al., Wie ticken Jugendliche 2016?,

DOI 10.1007/978-3-658-12533-2_10 
Die Identitätsentwicklung gilt als zentrale Aufgabe in der Adoleszenz - Selbstfindung ist das Thema des Jugendalters. Neben individuellen biografischen Merkmalen, die sich zu einer einzigartigen Persönlichkeitsstruktur verdichten, spielen dabei auch soziale Leitbilder und Gruppenzugehörigkeiten eine Rolle. In diese Kategorie fällt v. a. auch die Nationalität. Umgekehrt sind Zugehörigkeitsgefühle und eine emotionale Identifikation auch wesentliche Bausteine nationaler Identität.

Die in der vorliegenden Untersuchung dazu gestellte Forschungsfrage war, welchen Zusammenhang es heute, in einer globalisierten und international vernetzen Welt, zwischen nationaler und persönlicher Identität gibt. Ist die Nationalität bei den heutigen Jugendlichen ein relevanter Aspekt des Selbstbilds und der persönlichen Identität? Speziell für die Einwanderungsgesellschaft Deutschland schließt sich die Frage an, wie eine solche nationale Identität heute aussehen könnte. Fühlen sich die 14- bis 17-Jährigen in der Lage, eine deutsche nationale Identität zuzulassen, oder ist für sie vor dem Hintergrund der deutschen Vergangenheit eine ungebrochene Identifikation nach wie vor schwierig? Wird die neue Vielfalt des Landes, in dem Migration zum Normalfall geworden ist, von den Jugendlichen akzeptiert - und hat sich in der jungen Generation evtl. bereits ein entsprechendes neues Leitbild entwickelt?

Neuere Erhebungen zeigen, dass die überwiegende Mehrheit der Deutschen sich wieder positiv zur eigenen Nation bekennt. Und es zeigt sich auch, dass eine Mehrheit unter den Deutschen mit Migrationshintergrund in Umfragen angibt, sich "deutsch" zu fühlen. ${ }^{6}$ In solchen Erhebungen wird häufig gefragt, wie sehr man sich als Deutscher bzw. Deutsche fühle. Aber was heißt das überhaupt - "Deutsch-Sein"? Das haben wir in den mit den Jugendlichen geführten offenen Interviews exploriert. Wir wollten wissen, woran sich Deutsch-Sein im Jahr 2015 festmacht und was sich aus Sicht der Jugendlichen hinter den Begriffen Nation und Nationalität verbirgt. Worin manifestieren sie sich, und wo werden sie erlebbar? Was ist eigentlich heute "typisch deutsch" und worauf könnte ein positives Nationalbewusstsein - sollte es denn vorhanden sein - beruhen?

6 Vgl. Naika Faroutan et. al. (2014) Deutschland postmigrantisch I. Gesellschaft, Religion, Identität. Erste Ergebnisse. Online über: https://www.projekte.hu-berlin.de/de/junited/deutschland-postmigrantisch-1/ 
Speziell mit Blick auf Jugendliche mit einem Migrationshintergrund war die Frage, ob es ihnen gelingt, ethnische und nationale Identität miteinander zu verknüpfen - und ob sie dieses Ziel überhaupt haben. Lars Leszczensky und Aitana Gräbs Santiago über dieses Verständnis "doppelter Identität“ von Migranten: „Ethnische Identität besteht dabei aus einem Wissen um die eigene ethnische Herkunft, mit dieser Herkunft verbundenen Bewertungen und einer emotionalen Bindung an die Herkunftsgruppe. Nationale Identität ist aus Sicht von Migranten das Gegenstück zur ethnischen Identität und hat als Bezugspunkt das sogenannte Aufnahmeland. Diese Identitätsformen schließen sich nicht aus, sondern ergänzen sich in einem Idealfall, den wir als Integration beschreiben, gegenseitig. Man spricht, wenn eine Identifikation sowohl mit dem Herkunfts- als auch mit dem Aufnahmeland besteht, von dualen Identitäten. ${ }^{17}$

Vergleichbar damit ist das Konzept der hybriden Identität ${ }^{8}$ von Menschen, die sich zwei oder mehreren kulturellen Räumen gleichermaßen zugehörig fühlen.

\section{Verschiedene Assoziationen zu den Begriffen Nation und Nationalität.}

Die nachstehende Grafik listet die häufigsten spontanen Assoziationen der befragten Jugendlichen zum Begriffspaar "Nation/Nationalität" auf. Dabei zeigt sich ein breites und durchaus heterogenes Bedeutungsfeld mit Nennungen unterschiedlichster emotionaler Aufladung.

\section{Die nationale Identität ist den meisten (v. a. autochthonen) Jugendlichen nicht besonders wichtig.}

Bittet man die 14- bis 17-Jährigen, spontan zu den Begriffen „Nation“ und "Nationalität" zu assoziieren, zeigt sich, dass diese zunächst nicht notwendigerweise negativ besetzt sind. Häufig nehmen die Jugendlichen gar

\footnotetext{
7 Lars Leszczensky \& Aitana Gräbs Santiago (2014) Die Messung ethnischer und nationaler Identität von Kindern und Jugendlichen. Arbeitspapiere - Working Papers, Nr. 155, 2014, Mannheimer Zentrum für Europäische Sozialforschung. Online über: http://www.mzes.uni-mannheim.de/publications/wp/wp-155.pdf.

8 Vgl. Das Forschungsprojekt „Hybride Identitäten in Deutschland” an der FU Berlin - www.heymat.hu-berlin.de
} 


\section{Assoziationen zu den Begriffen „Nation / Nationalität"}

\section{Verschiedenheit / Gleichheit sprache}

Staatsangehörigkeit

\section{Herkunft}

Vielfalt

Traditionen

\section{Patriotismus}

Deutschland

Frieden
Urlaub

Identität

Vaterland

\section{Vorurteile}

Nationalstolz
Zugehörigkeit

Eigenes Herkunftsland

Museen, Denkmäler, Gedenkstätten

\section{Land / Länder Abgrenzung}

keine Wertung vor und verweisen beispielweise beim Begriff "Nationalität" auf seine lexikalische Bedeutung im Sinne von „Herkunft". Allerdings zeigt sich: je höher das formale Bildungsniveau und je postmoderner die Grundorientierung ist, desto wahrscheinlicher wird eine kritische Deutung der Begriffe. Direkte Bezüge zum Nationalsozialismus, zu Rassismus oder Rechtsextremismus stellen entsprechend vor allem bildungsnahe Jugendliche aus dem postmodernen Segment her. Sie grenzen sich teilweise auch ganz bewusst von jeder Form des Nationalstolzes ab und betonen, dass Nationalität schließlich nicht auf persönlichen Leistungen beruht, sondern qua Geburt erworben wird.

Auffällig ist, dass sich vor allem bei Adaptiv-Pragmatischen kein konsistentes Begriffsbild mit einer eindeutigen bewertenden Tendenz zeigt. Sie verweisen sowohl auf positive Assoziationen rund um Traditionen, wie Weihnachten oder landestypische Spezialitäten, als auch auf gedankliche Verbindungen, die sich auf ein gesteigertes Nationalbewusstsein und damit einhergehende Gefahren beziehen.

Die Jüngeren können mit "Nation" oder "Nationalität" teils (noch) wenig oder gar nichts anfangen. Weder die begriffliche Bedeutung, noch der 
Diskurs um eine historische "Vorbelastung" der Begriffe sind ihnen geläufig.

( Ich finde, man kann auf seine Herkunft stolz sein, wenn man möchte. Man kann auch sagen, ich komme daher und vertrete dieses Land. Mir sind Traditionen jetzt nicht wichtig, aber bestimmte Traditionen, die im Land verankert sind, die werde ich auch pflegen - selbst wenn ich mal wegziehen sollte aus Deutschland. Ich werde Weihachten so feiern, wie es in Deutschland ist. Aber ich finde es trotzdem spannend, mir diese anderen Traditionen anzugucken. Aber ich finde, man darf es auch nicht übertreiben und sagen, mein Land ist besser als deins. Ja, man kommt da her aus dem Land, man ist da geboren, man hat diese Traditionen, und das ist auch schön und gut, aber es ist kein Land der Welt besser als ein anderes. (weiblich, 17 Jahre, kein Migrationshintergrund, Adaptiv-Pragmatische)

8 [INT: An WAS DENKST DU, WENn Du dAS WORT "NATION" Oder "NATIONAlitäT" hÖRSt?] Deutschland. [INT: Warum DeUtschLAND?] Ich weiß es nicht. Ich glaube, durch die Nachrichten. Ich habe irgendwas von Nationalität und Deutschland gehört. Ich glaube, das ist noch hängengeblieben. (weiblich, 14 Jahre, kein Migrationshintergrund, Adaptiv-Pragmatische)

( Das heißt einfach, aus welchem Land man kommt. Aber mehr fällt mir nicht dazu ein, nein. (weiblich, 14 Jahre, Migrationshintergrund, Expeditive)

[INT: UND AN WAS DENKST DU, WENN DU DAS WORT ,NATION' ODER 'NATIONAlitÄT' HÖRST?] Keine Ahnung, an gar nichts. (weiblich, 14 Jahre, Migrationshintergrund, Prekäre)

Nation? Rassismus. Das wäre das Erste, woran ich denke. (männlich, 17 Jahre, Migrationshintergrund, Konservativ-Bürgerliche)

( Da denke ich natürlich direkt an Hitler, muss ich ganz ehrlich zugeben. Das verbindet man halt damit. Gerade, weil Nationalität...nein, eigentlich hat es nicht wirklich was mit Hitler zu tun. Also doch, ja. Aber man muss dann nicht gleich Hitler damit verbinden. [INT: ABER DAS IST SO 
Deine erste Assoziation?] Ja. (weiblich, 16 Jahre, kein Migrationshintergrund, Sozialökologische)

( Naja, wo Menschen herkommen. Das ist Nationalität, das bedeutet es für mich. [INT: SPIELT ES FüR DICH EINE ROLLE, WO JEMAND HERкоммт?] Nein, es spielt keine Rolle für mich. (weiblich, 15 Jahre, Migrationshintergrund, Materialistische Hedonisten)

Jenseits der Verbindung der Begriffe "Nation/Nationalität" mit dem Nationalsozialismus ist in den spontanen Assoziationen der Jugendlichen in Umrissen das neue, kontrovers in der Migrationsforschung diskutierte Konzept und die Narrative der "postmigrantischen Gesellschaft" erkennbar. Die Äußerungen zu "Herkunft", „Verschiedenheit" und „Vielfalt" nehmen jedenfalls auf die durch Migration veränderte Situation im Land ausdrücklich Bezug.

\section{Eigene Erfahrungen mit rechtsextremen Denkmustern werden selten berichtet.}

Persönliche Erfahrungen mit Rechtsextremismus oder Neonazismus, nationalsozialistischem oder rassistischem Gedankengut im Freundes- oder Bekanntenkreis kommen in den Interviews kaum zur Sprache. Lediglich einige Befragte aus den ostdeutschen Bundesländern berichten von Auseinandersetzungen mit entsprechenden Bekenntnissen innerhalb ihres Freundeskreises.

Man sagt ja "Die deutsche Nation". Ich weiß nicht so, aber ganz schön viele Freunde von mir sind so total auf Nazitrip, wo ich immer sage: Ey Alter, Mann, du bist kein Nazi, hör auf mit dem Scheiß! Und der so: Doch, ich bin Nazi! Ich sage immer, Nazi sein ist nicht cool sein. Ich finde es scheiße. Ich sage: Wenn du Nazi bist, brauchst du nicht mit mir reden, dann kannst du da hinten mit der Wand reden. Hitler hat eine gute Sache gemacht, und zwar die Autobahn. Das ist das Erste, was mir einfällt, Autobahn. (weiblich, 14 Jahre, kein Migrationshintergrund, Experimentalistische Hedonisten, Ostdeutschland) 
(1) [int: Fallen Dir irgendwelche Symbole ein zUm Stichwort "DEUTSCH" ODER „DEUTSCH SEIN"?] Das sind die Symbole, die die Nazis gerne benutzen, also Hakenkreuz oder irgendwelche deutschen Abzeichen. Was ja eigentlich mehr oder weniger verboten ist. Und den Leuten sieht man dann wirklich an, welchen Hass die auf Ausländer haben. Und es gibt hier auch genug davon in der Stadt, wo man es schon aus der Musik heraushört. Also es gibt viele, die so sind, also solche Verrückten und Bekloppten. (männlich, 15 Jahre, kein Migrationshintergrund, Materialistische Hedonisten, Ostdeutschland)

\section{Die ethnische Identität fördert bei Jugendlichen mit Migrationshintergrund Vergemeinschaftung.}

Die Untersuchungsbefunde zeigen, dass Jugendliche mit Migrationshintergrund - insbesondere aus dem traditionellen und dem modernen Segment - tendenziell eine positivere Haltung gegenüber den Begriffen Nation und Nationalität haben. Sie stellen in der Regel keine historischen Bezüge zum Nationalsozialismus oder anderen Ausformungen übersteigerten Nationalbewusstseins her, sondern denken vornehmlich an ihre eigene ethnische Herkunft, die ein wichtiger Identitätsanker und für viele - was unumwunden zugegeben wird - auch ein Quell des Stolzes ist.

Diese ethnische Identität wird in verschiedenen Situationen im Alltag positiv erlebt und ist häufig an einen sozialen Kontext gebunden - Familienfeste, gemeinsame Essen, unterwegs sein mit Freunden und ähnliches. Aber auch innerhalb dieser Gruppe werden beim Stichwort "Nation/ Nationalität" gelegentlich negative Konnotationen aufgerufen. Man beschreibt Diskriminierungs- und Exklusionserfahrungen, die, so die Einschätzung, auf Stereotypisierungen ethnischer oder religiöser Gruppen beruhen - auch wenn sie nicht allzu weit verbreitet seien und von der Mehrheit der Bevölkerung abgelehnt würden. 
Typische Zitate zur |llustration

Für mich ist Nationalität eine Sache, wo man sagen kann, ich bin der und der. Ich selber bezeichne mich natürlich als russisch. Aber beim Thema "Nationalität" kommen auch sofort Vorurteile in den Kopf. Es gibt Personen, die haben Vorurteile gegen bestimmte Nationalitäten. Und so etwas verbinde ich mit dem Begriff "Nationalität". Ich selber habe keine Vorurteile. Für mich ist jeder Mensch ein Mensch, egal welche Nationalität er hat. (männlich, 16 Jahre, Migrationshintergrund, Adaptiv-Pragmatische)

(7) [INT: GibT Es SitUATIONEN, IN DENEN DU DENKST: „DAS SIND WIR DeUTSCHE"?] Nein. [INT: UND GIBT ES SITUATIONEN, Wo DU DENKST: "DAS SIND WIR ITALIENER"?] Ja, wenn wir alle zusammensitzen, und es ist total laut. Und wir essen. Eben einfach die Familienfeste. (weiblich, 14 Jahre, Migrationshintergrund, Adaptiv-Pragmatische)

[INT: GIBT ES AUCH ETWAS TYPISCH JORDANISCHES?] Bei mir sind das in der Familie, der Haushalt und die Stimmung, das Essen. (männlich, 16 Jahre, Migrationshintergrund, Prekäre)

[INT: Und WENN DU DAS WORT NATION ODER NATIONALITÄT HÖRST, WORAN DENKST DU DA?] Stolz. Also manche Leute sind stolz auf ihre Nationalität und andere nicht. Also ich bin sehr stolz. Ich bin ja eigentlich halb Deutscher, weil ich hier geboren bin. Aber ich bin auch stolz, halb Italiener zu sein. (männlich, 16 Jahre, Migrationshintergrund, Materialistische Hedonisten)

[INT: GIBT ES IRGENDWIE, WAS DANN SO TYPISCH TÜRKISCH ODER SO IST? ODER TYPISCH ARABISCH, TYPISCH ITALIENISCH, TYPISCH SPANISCH?] Ja, eigentlich bei allen so gemeinsam, dass wir wirklich alle sehr an unsere Familie gebunden sind. Und auch mit unserer Familie sehr viel Zeit verbringen. Und dass wir auch sehr viel Besuch haben und sehr oft bei jemandem zu Hause sind. (...) Weil Familie immer groß ist und da reicht einem die Familie meistens. (weiblich, 16 Jahre, Migrationshintergrund, Konservativ-Bürgerliche)

( Ich habe zwei Nationalitäten. Ich bin stolz auf meine Länder. Klar gibt es hier und da politisch irgendwelche Fehler. Aber an sich bin ich auf meine Länder total stolz. (weiblich, 16 Jahre, Migrationshintergrund, Materialistische Hedonisten) 
( I I b bin ja in Berlin aufgewachsen. Also man hat ja immer so einen Migrationshintergrund, würde ich sagen. Man ist ja Deutscher sozusagen. Man ist ja hier geboren, aber hat halt sozusagen so einen türkischen Hintergrund, auch durch die Familie. Und da würde ich dann sagen, dass ich mich eher als Türke fühle, aber auch so ein bisschen deutsch. Das ist halt so ein bisschen kompliziert. (männlich, 16 Jahre, Migrationshintergrund, Materialistische Hedonisten)

\section{Bewusste Abgrenzung von der Nation als identitätsstiftendem Merkmal in bildungsnahen Lebenswelten.}

Vor allem die Sozialökologischen, Expeditiven und Experimentalistischen Hedonisten betonen, dass die Begriffe Nation und Nationalität historisch vorbelastet sind. Aus ihrer Perspektive sollten diese Begriffe positiv umgedeutet werden und Diversität und Vielfalt in Gleichheit beschreiben - wie sie fast mantra-artig antworten.

Kennzeichnend für die postmodernen Lebenswelten, allen voran die Expeditiven, ist zudem, dass man sich - und anderen Deutschen - keine bruchlose Identifikation mit dem Herkunftsland zugesteht. Ein Rückbezug (im Sinne von Rückzug) auf die nationale Identität scheint der internationalistischen bzw. globalen Grundorientierung dieser Lebenswelten von vornherein zu widersprechen.

Vor allem bei den Expeditiven und teils auch bei den Sozialökologischen ist das Bestreben erkennbar, sich von einer Vorstellung von nationaler Identität als auf äußere Merkmale bezogene Kategorie zu lösen. Sie scheinen fast erleichtert, wenn sie darauf verweisen, das Deutschsein heute nicht mehr mit bloßem Auge erkennbar sei. Auch die Fragen, die sich die jugendlichen Interviewer aus diesen Lebenswelten zum Thema „Deutschland“ überlegt haben, verdeutlichen die kritische Haltung gegenüber Nationalität als Identitätsbaustein und übersteigertem Patriotismus. 
Typische Zitate zur |llustration

Aber generell finde ich eigentlich dieses Nationalgefühl, generell von allen Leuten, ein bisschen lächerlich. Weil niemand kann was dafür, wo er geboren ist. Und ich finde, man kann auch nicht stolz darauf sein. Das ist halt so, wie es ist. Warum kann man denn stolz darauf sein, wo man geboren ist? Das hat man nicht selbst erreicht. Deswegen finde ich es generell eigentlich unnötig. (weiblich, 16 Jahre, kein Migrationshintergrund, Sozialökologische)

( Als ich in den USA war, war Nationalität, Nationalbewusstsein, vielleicht auch Patriotismus viel ausgeprägter als in Deutschland. In Deutschland merkt man das ja eigentlich echt nur bei irgendwelchen Weltmeisterschaften. Dass da dann irgendwelche Fahnen rausgeholt werden, aber in den USA ist es so, dass da an jeder zweiten Haustür eine hängt. Ich finde es aber auch gut, dass es in Deutschland nicht so ist. Wir haben so eine Vergangenheit. Ich meine, es ist Vergangenheit, aber es ist trotzdem irgendwie noch komisch. Ich bin da so ein bisschen skeptisch bei Patriotismus. (weiblich, 17 Jahre, Migrationshintergrund, Expeditive)

(3)i "Nation" oder "Nationalität" denke ich einfach an die Vielfalt von den ganzen Nationalitäten, kultureller Austausch. Ich weiß nicht, irgendwie fallen mir Gewürze ein von anderen orientalischen Gegenden und einfach auch die Urlaubsziele, wo wir schon öfter waren. Einfach generell die ganze Vielfalt von der Menschheit. Ja, da fällt mir halt Zweiter Weltkrieg ein, weil das war ja immer so, ein Volk, eine Nation und so. Das von heute ist natürlich positiv, aber so die Geschichte lastet uns ja teilweise immer noch so an. (weiblich, 16 Jahre, kein Migrationshintergrund, Sozialökologische)

\section{[INT: GIBT ES IRGENDWAS, WAS DU, TYPISCH DEUTSCH' FINDEST? WAS} UNS SO ALS DEUTSCHE VERBINDET ODER WO MAN ES SIEHT, AN WELCHEN GelegenHeIten?] Nee, nicht wirklich. Es gibt eigentlich ja auch Leute aus komplett anderen Ländern. Bei mir in der Arbeit, der kommt zum Beispiel aus dem Irak, und der sieht halt einfach pur aus wie ein Deutscher. Kommt glaube ich nicht drauf an, wie man aussieht. Es gibt ja auch so Asylbewerber, die so komplett aussehen wie Deutsche. (weiblich, 15 Jahre, kein Migrationshintergrund, Experimentalistische Hedonisten) 
Auszug aus einem Fragebogen, den eine Jugendliche zum Thema "Deutschland" entwickelt hat.

Deutschland:

Könntest du dir vorstellen später Deutschland zu verlassen und auszuwandern?

Wenn ja, welche Länder sind für dich attraktiver als Deutschland und weshalb?

Was zeichnet Deutschland/ Deutsche besonders aus? Was magst du? Was magst du nicht?

Wie ist das für dich, wenn die deutsche Nationalmannschaft Fußball spielt? Wie fühlst du dich dann?

Wenn du im Ausland bist, sagst du dann gerne, dass du aus Deutschland kommst? Wenn nicht:

Woran liegt das?

weiblich, 17 Jahre, Expeditive

Aber ich glaube, Deutschland ist zum Glück auch so ein bisschen kulturell durchgemischt, dass man nicht so den Stereotyp hat, wo man denkt: Der ist deutsch und alle anderen können nicht deutsch sein. (weiblich, 17 Jahre, Migrationshintergrund, Expeditive)

(1) Hautfarbe sagt nichts über Menschen aus. (weiblich, 17 Jahre, Migrationshintergrund, Experimentalistische Hedonisten, Berlin)

[INT: GIBT ES Für DICH ETWAS TYPISCh DeUtsches?] Nein, ich glaube nicht. Also, da kann man natürlich die Vorurteile sagen, aber für mich selber ist eigentlich nichts so typisch Deutsch. (weiblich, 16 Jahre, kein Migrationshintergrund, Expeditive)

\section{Die „Fußball-Nation Deutschland" ist nicht für alle Lebenswelten Identifikationsobjekt.}

Natürlich denken viele Jugendliche beim Stichwort "Nation" auch an Fußball, die Nationalmannschaft und die Fußballweltmeisterschaft.

(7INT: Und WAS DENKST DU ZUM BeISPIEL, WENN DU dAS Wort Nation oder Nationalität hÖRSt?] Da denke ich irgendwie an 
Fußball. (weiblich, 15 Jahre, kein Migrationshintergrund, Materialistische Hedonisten)

( $)$ Ähm ... Ich weiß nicht, was Nation bedeutet. Aber ... Ich habe gerade an das Wort «Nationalmannschaft» gedacht. (männlich, 15 Jahre, Migrationshintergrund, Materialistische Hedonisten)

( IINT: Gibt es so Situationen oder Momente, wo man so spürt, DAS SIND WIR DEUTSCHEN ODER SO ETWAS?] I ch weiß nicht, beim Sport, da ist man auf seine Nation ja stolz. Deutschland ist Weltmeister, wir sind Weltmeister. Das ist halt so. Das ist halt einfach so. Da merkt man auch, da gehört man zu Deutschland dazu. (weiblich, 17 Jahre, kein Migrationshintergrund, Adaptiv-Pragmatische)

(INT: GIBT ES IRGENDWIE SitUATIONEN ODER THEMEN, WO MAN DAS SO SPÜREN KANN, DASS MAN IRGENDWIE SAGT: ,JA, WIR DEUTSCHE'?] Auf jeden Fall auch bei der Fußball-WM, da. Und halt auch so auf so Volksfesten, zum Beispiel die Wies'n oder so, ist halt klar, ist halt bayrisch. Aber sonst nicht, nee. (weiblich, 17 Jahre, kein Migrationshintergrund, Materialistische Hedonisten)

Spontane Äußerungen zu diesem Komplex sind zwar weniger häufig, als man angesichts der noch nicht lange zurückliegenden WM vermutet hätte, bei entsprechenden Nachfragen wird aber deutlich, dass Fußball das Moment des Erlebbarwerdens nationaler Identität für den jugendlichen Mainstream ist - vor allem für Jugendliche ohne Migrationshintergrund. Vereinzelt klingt im Zusammenhang mit diesem Thema auch Kritik daran an, dass ein Nationalbewusstsein und erst recht Nationalstolz in Deutschland nicht unbefangen geäußert werden können.

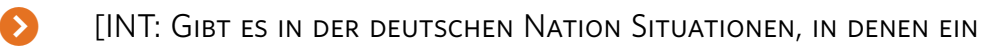
WIR-GEFÜHL ENTSTEHT?] In der deutschen Nation nicht. Weil alle, die nicht rechts sind, gerade versuchen, das zu vermeiden. Man muss ja hier wirklich aufpassen, was ärgerlich ist und was auch nervt. Weil gerade beim Fußball zum Beispiel, da wird man als Deutscher auch schnell als Nazi abgestempelt. Und das ist natürlich ärgerlich, dass man da nicht so dafür sein kann. Also jetzt nicht für Deutschland, sondern für das 
Fußballspiel zum Beispiel. (weiblich, 16 Jahre, kein Migrationshintergrund, Konservativ-Bürgerliche)

Sensibler zeigen sich auch beim Thema Fußball die postmodernen Lebenswelten sowie die Sozialökologischen. Dabei lassen sie durchaus auch ein Unwohlsein erkennen, wenn sie sich als Deutsche nicht vorbehaltlos zur eigenen Mannschaft und damit zur eigenen Nation bekennen. Vereinzelt wird von den befragten Jugendlichen auch die ethnische Vielfalt der deutschen Nationalmannschaft als Hinweis für ein neues, diverses Deutschland und ein ethnisch facettenreiches "Deutsch-Sein" herangezogen.

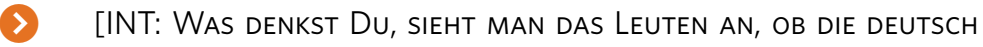
SIND ODER NICHT?] Na ja, ich meine, es gibt ja auch Fußballer, die sind zum Beispiel jetzt dunkelhäutig, denkst Du ja eigentlich auch, dass die aus Ghana oder aus Brasilien oder so kommen und dabei haben sie einen deutschen Namen wie Antonio Rüdiger. Der ist ja auch deutscher Nationalspieler oder Jérôme Boateng. Ich meine, Jérôme ist jetzt nicht so der typisch deutsche Name, aber der ist ja auch ein Deutscher. (männlich, 14 Jahre, kein Migrationshintergrund, Konservativ-Bürgerliche)

\section{Staatsbürgerschaft und Nationalsymbole haben wenig Bedeutung.}

Ihre Staatsbürgerschaft ist für die befragten Jugendlichen kein lebendiges Merkmal ihrer Identität. Der Pass wird als Ausweis der Aufenthaltsberechtigung gesehen, teilweise auch der Herkunft - selten aber als Symbol der nationalen Identität. Auch zu Nationalsymbolen (wie beispielweise Flagge, Hymne, Bundesadler) haben die Jugendlichen in der Regel keinen emotionalen Bezug.

So wird bei den spontanen Assoziationen zum Begriff "Nation" die Nationalhymne lediglich ein einziges Mal genannt. Auf Nachfragen wird deutlich, dass diese Symbole kein Gegenstand von Identifikation oder gar Verehrung darstellen und zumindest für autochthone Jugendliche weitgehend irrelevant sind. Eine Ausnahme bilden Jugendliche mit 
Migrationshintergrund der unterschichtigen Lebenswelten. Hier finden sich teilweise auch entsprechende Symbole in den Jugendzimmern.

Typische

Zitate zur Illustration

So ein Pass ist einfach. Da ist es jetzt einfach so: Wenn man einen Pass hat, ist man halt auf dem Papier Deutscher, aber vielleicht ist man ja Nationalität Türke. Ich würde sagen, das spielt nicht so eine große Rolle. (männlich, 16 Jahre, Migrationshintergrund, Materialistische Hedonisten)

8 Wenn mir jemand sagt, dass er Spanier ist und trotzdem einen deutschen Pass hat, gehe ich davon aus, dass seine Familie aus Spanien kommt, also seine Eltern oder Großeltern. Und dass er vielleicht sogar die Sprache spricht. [INT: WelChe Rolle SPIELT DABEI DER PASS?] Am Pass kann man identifizieren, aus welchem Land ein Mensch kommt. (männlich, 15 Jahre, kein Migrationshintergrund, Adaptiv-Pragmatische)

(7) [INT: DU hast Ja HIER AUCH EINE FlagGE DES Kosovo DORT HÄNGEN. IST DIR DAS WICHTIG, SO ALS IDENTIFIKATION MIT DEM LAND?] Ja, das schon ja, ich bin stolz auf mein Land. Weil ich halt aus dem Land komme. (männlich, 14 Jahre, Migrationshintergrund, Prekäre)

(1) [INT: WAS DENKST DU DENN, WAS MACHT DENN JEMANDEN ZU EINEM DeUTSCHEN?] Wenn er in Deutschland geboren ist. (männlich, 14 Jahre, kein Migrationshintergrund, Konservativ-Bürgerliche)

\section{Stereotype Vorstellungen über andere Nationen/Kulturen sind weit verbreitet.}

Wir wollten von den Jugendlichen wissen, wie präsent nationale bzw. ethnische Identität in ihrem Alltag ist und haben in diesem Zusammenhang nach „typisch Deutschem" bzw. nach etwas Typischem für andere Nationalitäten gefragt. Dabei wurden bewusst verschiedene Nationalitäten als Beispiele genannt. Die Frage im Interview lautete: Woran erkennt man im Alltag, dass jemand deutsch, türkisch, russisch, italienisch usw. ist? 
Insgesamt reichen die Beschreibungen der Befragten von eher harmlosen Klischees bis hin zu harten Vorurteilen und diskriminierenden Verallgemeinerungen. Viele Jugendliche reflektieren durchaus, dass es sich dabei um rassistische Vorurteile handelt, und äußern auch ihr Unbehagen, von diesen beeinflusst zu sein. Sie scheinen nach alternativen Definitionen und Deutungsmustern zu suchen. Insbesondere bei den jüngeren und formal niedrig gebildeten Jugendlichen finden sich häufig naiv anmutende Stereotypisierungen ethnischer Gruppen, die aber nicht den stark abwertenden Charakter manifester Vorurteile haben. Am häufigsten finden sich diskriminierende Charakterisierungen bei Adaptiv-Pragmatischen.

(1) Die Deutschen sind meistens blond, blaue Augen. Die meisten Dunkelhaarigen sind eher Ausländer, aber sonst eigentlich nicht so. (männlich, 15 Jahre, Migrationshintergrund, Materialistische Hedonisten)

Typische Zitate zur Illustration

Bei den Italienern, die haben ja immer viel Temperament. Die Amis sind halt teilweise ein bisschen einfältig. Die Franzosen sind vielleicht ein bisschen zurückgeblieben, sagt man manchmal. Spanier sind auch so lebensfreudig. Engländer sind immer so die Gastfreundlichen. Ja, weiß nicht. (weiblich, 17 Jahre, kein Migrationshintergrund, Sozialökologische)

(7INT: GIBT ES ETWAS TYPISCH DeUTSChes?] Natürlich gibt es etwas typisch Deutsches. Es gibt auch typisch Russisches. Typisch deutsch sind zum Beispiel Spätzle, Schnitzel, Kartoffelsalat. [INT: UND TYPISCHE VeRHALTENSWEISEN?] Es gibt dieses Schwäbisch, dass man halt sehr ehrgeizig ist. Aber für mich sind das teilweise auch Vorurteile. Man hat das zwar mal irgendwann so gehört, aber ich könnte am Verhalten nicht erkennen, welche Nationalität jemand hat. [INT: ALso so RICHTIG STIMMT DAS NICHT?] Nein. Man kann zwar sagen, die Russen trinken viel. Das ist auch so, das stimmt ja auch. Aber man kann nicht sagen, wenn einer auf einer Party zu viel trinkt: typisch Russisch. Das kann man nicht in Verbindung bringen. (männlich, 16 Jahre, Migrationshintergrund, Adaptiv-Pragmatische) 
(8) Wenn man irgendwie sehr gebildet redet, dann merkt man typisch Deutsch. Aber das ist ja nicht negativ. (männlich, 16 Jahre, Migrationshintergrund, Materialistische Hedonisten)

(7INT: GIBT ES DENN ETWAS TYPISCh DeUTSCHES?] Ja, klar. So etwas wie Pünktlichkeit wird uns ja immer nachgesagt. Obwohl das jetzt auf mich nicht wirklich zutrifft. Oder fleißig sein. Aber das sind ja auch nur Klischees. Also ich persönlich sehe da jetzt nicht irgendwas drin, was da stimmt. [INT: WENN DU HÖRST, JEMAND IST ZUM BEISPIEL TÜRKISCH, MACHT DAS DANN IRGENDWIE EINEN UNTERSCHIED?] Ja, jeder hat Vorurteile. Das ist so und das kriegt man leider auch nicht raus aus dem Kopf. Wenn ich an Amerikaner denke, dann denke ich direkt an Fettleibigkeit und Burger King. Das ist natürlich frech, aber jeder Mensch hat Vorurteile. Das ist nun mal so. Und wenn ich an Türken denke, dann denke ich oft an Player. So welche, die gerne Mädels anmachen, weil das einem selbst natürlich oft passiert ist. Das soll jetzt aber nicht abwertend sein. Aber es ist halt das, was man damit verbindet. (weiblich, 16 Jahre, kein Migrationshintergrund, Sozialökologische)

(7) Aber man sieht es auch meistens an dem Aussehen, also wie die Haare sind. So wenn es halt eher ein Schwarzkopf ist, dann weißt du halt, Araber oder Türke oder irgendwie sowas in der Art. Man kann das schon leicht so vom Aussehen kann man das schon erkennen. (männlich, 16 Jahre, kein Migrationshintergrund, Adaptiv-Pragmatische)

Unabhängig von den Klischeevorstellungen über andere Kulturen sind die meisten Jugendlichen davon überzeugt, dass alle Menschen gleiche Rechte haben. Entsprechend dieser sozial erwünschten Norm werden in der Regel Unterschiede zwischen Menschen mit und ohne Migrationshintergrund bestritten. Eines der wenigen Merkmale, die mit der Herkunft in Verbindung gebracht werden, ist die Religion und gelegentlich auch die Sprache.

Typische

Zitate zur Illustration
Das ist eigentlich ganz ... Also ich finde das gar nicht wichtig, wo er überhaupt herkommt oder wer er ist. Weil das ist einfach ... Jeder Mensch ist gleich, sozusagen. Keiner ist einfach besser oder keiner ist 
einfach niedriger. (männlich, 16 Jahre, Migrationshintergrund, Materialistische Hedonisten)

( Für mich geht es eigentlich nur auf die Persönlichkeit so. Der Rest ist mir dann egal, ob der aus Afrika oder aus der Türkei kommt oder aus Spanien, England, das ist mir dann egal. (männlich, 16 Jahre, kein Migrationshintergrund, Adaptiv-Pragmatische)

( Ich finde, es sind halt Menschen wie jeder andere. Ich würde da jetzt nicht direkt einen Unterschied machen. Das sind halt alles Menschen, das sind halt alles korrekte Leute. Ist halt so. Wenn die nett zu mir sind, bin ich nett zu denen. (männlich, 16 Jahre, kein Migrationshintergrund, Adaptiv-Pragmatische)

( Ich persönlich mache da keinen Unterschied. Wie gesagt, ich habe nicht so wahnsinnig viel mit solchen Leuten zu tun. Vielleicht auch weil ich auf einem humanistische Gymnasium bin, und sich das keiner antun will. (weiblich, 17 Jahre, kein Migrationshintergrund, Adaptiv-Pragmatische)

( Die Welt gehört ja eigentlich zusammen. Und wenn man dann sagt, der eine gehört hier gar nicht her, also der kommt von einem anderen Land und darf hier gar nicht rein, das stimmt halt gar nicht. Weil die Menschen dürfen und sollen sich auf der Welt so bewegen, wie sie selber wollen. (männlich, 16 Jahre, Migrationshintergrund, Materialistische Hedonisten)

( Es gibt ja schon Verhaltensunterschiede in den unterschiedlichen Ländern. Allein schon, wie man an eine Religion herangeht. Deutschland ist ein Land, in dem es relativ egal ist, ob man eine Religion hat oder nicht. Aber es gibt ja auch andere Länder, in denen Religion selbstverständlich ist. (weiblich, 15 Jahre, kein Migrationshintergrund, Expeditive)

Das positive Bild einer pluralen Zivilgesellschaft ist bei vielen Jugendlichen noch nicht gefestigt und selbstverständlich präsent. Ausnahme sind hier die weltgewandten Sozialökologischen und die postmodernen Gruppen, die nicht zuletzt auch mit der Aufnahme und Integration von Flüchtlingen die Hoffnung auf ein "bunteres Deutschland" verknüpfen (siehe auch Kapitel zu Flucht und Asyl). 
Erwähnt werden muss auch, dass bei manchen eine kritische Reflektion von landläufigen Stereotypen ausbleibt. Es gibt dabei Stimmen, die recht radikal eine konsequente Anpassung der Fremden an die deutsche Kultur - Assimilation - einfordern. Sie bleiben jedoch in der Minderheit, am ehesten tauchen sie noch im prekären und bürgerlichen Segment (Adaptiv-Pragmatische, Konservativ-Bürgerliche) auf (siehe auch Kapitel zu Geflüchteten). Auch Negativklischees gegenüber bestimmten ethnischen und religiösen Gruppen finden sich vor allem im bürgerlichen Segment.

( Türkischen ... Ich nenne es jetzt mal Kanaken. Die gelen sich halt die Haare hinter, ziehen Lederjacken an und sind halt ein bisschen aggressiver unterwegs, sagen wir es mal so. Und da sieht man eigentlich, dass sie zum Beispiel türkisch, serbisch oder sowas sind. (weiblich, 15 Jahre, kein Migrationshintergrund, Adaptiv-Pragmatische)

( Ja, wie schon gesagt, also Respekt haben und dass sie einfach dann auch nicht so große Klappe haben, weil sie sind ja eigentlich nur zu Gast. Und ich meine ich gehe jetzt zum Beispiel auch nicht nach Spanien und habe dann eine große Klappe, da benehme ich mich auch ganz normal, dann sollen die sich hier eigentlich auch normal benehmen können. (männlich, 14 Jahre, kein Migrationshintergrund, Konservativ-Bürgerliche)

Es gibt allerdings auch Jugendliche, die an dieser Stelle auf strukturelle Benachteiligung und Diskriminierung von Menschen nicht-weißer Hautfarbe, nicht-deutsch klingender Namen oder ohne perfekte Sprachkenntnisse, beispielsweise auf dem Arbeitsmarkt, verweisen. Ein Bewusstsein für realen Alltagsrassismus und Diskriminierungstatbestände ist demnach durchaus vorhanden - allerdings eher bei den formal höher gebildeten Jugendlichen.

Typische Zitate zur Illustration

( Deswegen, bestimmt bei so Sachen, wo es dann um die Deutschkenntnisse geht oder im Beruf, wenn man dann gebrochen Deutsch spricht, dann ist es bestimmt nicht so von Vorteil. Aber mir würde es glaube ich genauso gehen, also ... [INT: UND EINFACH WIRKLICH AUCH GANZ ALLGEMEIN, MEINST DU, DAS SPIELT EINE Rolle BEI EINER BEWERBUNG, OB DA 
DRAUF STEHT IRGENDWIE "AHMET" ODER "HANS"?] Nee, ich glaube ... Eigentlich sollte es ... Es sollte keine Rolle spielen, aber in manchen Firmen spielt es bestimmt noch eine Rolle. Also ... Denke ich mir mal. (weiblich, 17 Jahre, kein Migrationshintergrund, Sozialökologische)

Also vom Prinzip her sollte es eigentlich nicht unbedingt eine Rolle dabei spielen, nur denke ich mal, dass bei bestimmten Dingen eher ein Deutscher angenommen wird als ein Ausländer. (männlich, 16 Jahre, kein Migrationshintergrund, Adaptiv-Pragmatische)

( Wenn man jetzt als ein Türke sich zum Beispiel für eine Anstellung bewirbt und da kommt ein Deutscher, da wird es wahrscheinlich einen Unterschied geben. (männlich, 17 Jahre, Konservativ-Bürgerliche)

\section{Vor allem die Jugendlichen mit einem türkischen oder arabischen Migrationshintergrund werden stigmatisiert.}

Türkeistämmige Deutsche und in Deutschland lebende türkische Staatsbürger gelten aus Sicht der heute 14- bis 17-Jährigen häufig als "die Ausländer" schlechthin. „Die Türken und Araber" sind die Gruppen, von denen man sich am häufigsten abgrenzt und die sich entsprechend auch selbst häufig von den einheimischen Jugendlichen abgrenzen. Gerade gegenüber "den Türken" bestehen zahlreiche Klischees und Vorurteile. So sind beispielsweise das Kopftuch bei Mädchen und das aggressive Auftreten bei Jungen für viele Jugendliche typische Merkmale des "Türkisch-Seins". Dabei geht es nicht um generalisierte Feindschaften zwischen Großgruppen, sondern um Rangeleien und Auseinandersetzungen auf dem Schulhof und auf der Straße. Derlei Situationen schildern Jugendliche mit und ohne Migrationshintergrund. Lebensweltlich zeigen sich dabei Schwerpunkte bei den Materialistischen Hedonisten, Prekären und teils auch bei den Adaptiv-Pragmatischen.

(1) Also es gibt ja auch diese Orte zum Beispiel so vor McDonalds und sowas. Und da sagen eigentlich alle: "Okay, die Türken." Also ich würde jetzt auch nicht sagen, dass das alles Türken sind, aber das ist Typische Zitate zur Illustration halt oft so, dass die ziemlich aggressiv sind. Und dass die Deutsche in 
Deutschland als Deutsche beleidigen. [INT: WAS IST NOCH SO TYPISCH FÜR DIE, WENN DU SCHON KLISCHEEMÄSSIG VON DER GRUPPE SPRICHST?] Aggressiv. Oberflächlich vor allem. (männlich, 16 Jahre, kein Migrationshintergrund, Adaptiv-Pragmatische)

(8) Kopftücher tragen. Da würde ich sagen: typisch türkisch. (weiblich, 15 Jahre, kein Migrationshintergrund, Materialistische Hedonisten)

( Ja. Also man sieht ja, wenn eine Frau zum Beispiel Türkin ist, trägt sie ein Kopftuch, manchmal. Nicht immer, aber meistens. Deutsche Frauen tragen auch keine Kopftücher, und ich finde, dass die, wenn die in unser Land kommen, dass die sich an unsere Vorschriften halten sollten, oder sich an uns anpassen sollten. Und ich verstehe nicht, warum türkische Frauen in Deutschland Kopftücher tragen. Darüber rege ich mich aber sehr lange schon auf. Ich habe Angst vor Menschen mit Kopftuch. Man sieht halt auch oft in den Nachrichten oder auf Facebook-Videos, wie Türken andere zusammenschlagen und ich habe auch ehrlich gesagt ein bisschen Angst vor den Menschen. (weiblich, 17 Jahre, kein Migrationshintergrund, Materialistische Hedonisten)

ICh finde Türken, allgemein so Muslime sind aggressiver als die Deutschen. Die wollen immer alle sofort schlagen, wenn jemand irgendwas Falsches sagt oder so was, was ja eigentlich gar nicht so gemeint ist. Und deswegen mache ich auch Kampfsport, damit ich mich auch wehren kann. Eigentlich konnte ich mich bisher immer wehren. [INT: Hattest Du selber auch SChON so KonflikTe?] Ja, sehr oft. An der Schule und auch außerhalb. Zum Beispiel: Ich bin jetzt mal an jemandem vorbei gelaufen, ganz normal, habe mich mit einem Kumpel unterhalten, der läuft direkt auf mich zu und rempelt mich an und sagt: Was soll denn das? Da habe ich dann gefragt: Was soll denn das von dir? Und dann sind wir halt in einen Konflikt geraten, und dann gab es halt so eine kleine Schlägerei. Und das tat mir in dem Moment auch gar nicht leid. Die zeigen ja einen dann auch nicht an, weil sie wissen, dass sie was falsch gemacht haben. (männlich, 15 Jahre, kein Migrationshintergrund, Prekäre)

( Es sind nicht größtenteils nur Vorurteile, dass Türken manchmal so ein bisschen ... Ich sehe das öfters in der Eisenbahnstraße, da fahre ich auch 
manchmal durch, da hat man schon ein mulmiges Gefühl. Da soll es auch ab und zu mal ein paar Schlägereien geben. (männlich, 14 Jahre, kein Migrationshintergrund, Adaptiv-Pragmatische)

Zum Beispiel verhalten sich Araber manchmal so richtig schlecht. Man merkt auch, wenn die schreien. Und die reden oder schreien auch manchmal auf Arabisch irgendwas. So wie die, die hier immer Stress machen. Das sind alles Araber. (männlich, 15 Jahre, kein Migrationshintergrund, Adaptiv-Pragmatische)

Vereinzelt werden von den Jugendlichen Stigmatisierungen von Muslimen geäußert. Ein dezidiert islamfeindliches Weltbild ist allerdings für keine Lebenswelt oder soziodemografische Gruppe per se typisch. In unserer Studie zeigen sich nur vereinzelt anti-islamische Ressentiments bei einem Teil der Adaptiv-Pragmatischen, Prekären und Materialistischen Hedonisten. In allen Lebenswelten ist die Abwehr solcher Stereotype aber deutlich größer.

Die Vorurteile gegenüber türkisch- und arabischstämmigen Jugendlichen sind einer der Gründe, weshalb diese Gruppen sich oft unter ihresgleichen befreunden. Zentrale Vergemeinschaftungsmerkmale Türkischund Arabischstämmiger sind zudem die gemeinsame Religion und Kultur, wobei Distinktionsbestrebungen auch sprachlich zum Ausdruck kommen ("wir" vs. "die"). Das heißt jedoch nicht, dass diese Jugendlichen keine andersstämmigen oder -gläubigen Freunde haben. Freunde mit der gleichen Religion sind ihnen allerdings näher. Oft werden auch Familienmitglieder, wie Cousins und Cousinen, als enge Freunde beschrieben.

(8) Wenn sie uns sehen, wir sind ja alles Ausländer, alles Türken in meinem Freundeskreis. (männlich, 17 Jahre, Migrationshintergrund, Materialistische Hedonisten)

Typische Zitate zur Illustration

[INT: Wie ISt ES JETZt ZUM BeISPIEL IN DeInem Freundeskreis, Sind DA NUR MOSLEMS, ODER SIND DA AUCH ANDERE RELIGIONEN VERTRETEN?] Ja, christlich oder so. Aber die meisten sind Moslems. (weiblich, 14 Jahre, Migrationshintergrund, Prekäre) 
(7INT: Sind die meisten Deiner Freunde Moslems?] Ja. Nein, ich glaube, zwei bis drei nicht, aber sonst, der Rest ja. Ich habe da eigentlich auch deutsche Freunde, mit denen ich auch befreundet bin. Aber nicht so intensiv wie mit den anderen. [INT: UND WAS GLAUBST DU, WORAN LIEGT DAS?] Ich glaube, sich zu verständigen, einfach so. (männlich, 16 Jahre, Migrationshintergrund, Materialistische Hedonisten)

( J) Ja, ich habe da eigentlich gar nichts gegen die Deutschen und meine Freunde auch nicht so. Eigentlich ist es uns einfach egal. [INT: ABER WIE KOMMT ES, DASS DU SO WENIG DEUTSCHE FREUNDE HAST?] Ich weiß nicht. Vielleicht liegt es auch daran, dass es einfach so ist. Dass ich mich eher mit meiner Kultur und auch mit den orientalischen Kulturen besser verstehe, anstatt mit den Deutschen. Ich weiß es nicht. (männlich, 16 Jahre, Migrationshintergrund, Materialistische Hedonisten)

(8) Bei manchen aus meiner Klasse ist das so, wenn du kein Türke bist, dann freunden sie sich gar nicht mir dir an. Das finde ich ein bisschen seltsam. Ich weiß gar nicht. Ich finde es auch komisch, dass die das gar nicht dann versuchen. Die haben, glaube ich, so ein schlechtes Bild von anderen Nationalitäten. (männlich, 16 Jahre, Migrationshintergrund, Adaptiv-Pragmatische)

\section{Bei Freundschaften spielt die Herkunft kaum eine Rolle, am wenigsten bei den soziokulturell modernen und bildungsnahen Milieus.}

Sehr viele Jugendliche haben Freunde mit einem Migrationshintergrund. Auffällig ist dabei, dass insbesondere in den soziokulturell modernen Milieus eine "postintegrative" Perspektive vorherrscht: Integration oder kulturelle Unterschiede sind für sie kein Thema, v. a. wenn die Freunde in Deutschland geboren und aufgewachsen sind.

Typische Zitate zur Illustration
Meine Freunde sind eigentlich alle deutsch. Also, ich hab eine Freundin, deren Vater kommt aus Afrika. Aber sie ist in Deutschland geboren. Sie ist schwarz, aber das nimmt man auch irgendwie gar nicht so wahr. Es ist halt eine Freundin, das ist halt nichts anderes. Eine Freundin von mir, 
ihr Vater kommt aus Spanien, aber sie ist in den USA geboren, aber lebt hier, also hat sie jetzt drei Pässe. (weiblich, 17 Jahre, Migrationshintergrund, Expeditive)

(1) Ja, also eine Freundin von mir, die ist Kongolesin, aber die ist schon mit zwei oder so hierher gezogen. Eine andere Freundin ist Russin, die ist auch mit zwei glaube ich hierher gezogen. (weiblich, 17 Jahre, kein Migrationshintergrund, Sozialökologische)

(In meinem Freundeskreis, die sind alle schon ganz, ganz früh, so mit zwei Jahren nach Deutschland gezogen. Meine beste Freundin ist zum Beispiel aus Thailand, aber die lebt hier auch schon seit sie zwei oder drei ist. Deswegen macht das eigentlich nicht so den Unterschied. (weiblich, 17 Jahre, Migrationshintergrund, Experimentalistische Hedonisten)

(I) In unserer Schule oder in meiner Klasse sind auch viele Leute, die einen französischen, serbischen oder griechischen Hintergrund haben. Ein Kumpel von mir ist auch halb Türke und halb Nigerianer. Also es gibt da einen kulturellen Mix. (männlich, 16 Jahre, Expeditive)

( Mein bester Freund ist Halbtürke. Sein Vater wohnt halt auch in der Türkei, aber er ist hier geboren, hier aufgewachsen. (weiblich, 16 Jahre, kein Migrationshintergrund, Experimentalistische Hedonisten)

( Bei meinen Freunden ist mir das vollkommen egal, wo die herkommen. Ich habe so viele Freunde, und die sind alle irgendwie Halb-Indonesier, Halb-Russen, Halb-Türken. Das ist mir vollkommen egal. [INT: Merkst Du da trotzdem kulturelle Unterschiede oder dass da trotzdem irgendwas anders ist?] Bei den meisten merkt man das eher weniger, gerade wenn wir unterwegs sind, weil die in Deutschland aufgewachsen sind. (weiblich, 17 Jahre, Migrationshintergrund, Adaptiv-Pragmatische)

( Ich habe alles Mögliche an Kollegen, Dunkelhäutige, ganz Helle, Albinos. Ich habe zwei Albinos, aber die sind halt auch mit Migrationshintergrund und aus Afrika, da habe ich Nordafrikaner, so etwas wie Marokko, Iran, Syrien. Viele Bekanntschaften, aber enge Freunde sind halt aus Mittelafrika, Nordafrika und Mitteleuropa, die ganzen Nachbarländer um Deutschland. Franzosen habe ich. Meine Freundin ist 
selber Portugiesin, ihr Vater ist Deutscher, aber irgendwie auch halber Schweizer oder Österreicher. (männlich, 16 Jahre, kein Migrationshintergrund, Materialistische Hedonisten)

Ja, na zum Beispiel wir haben in der Klasse zwei Portugiesen ... Portugiesinnen, zwei Mädchen ... Und mit denen, also mit der einen, mit der treffe ich mich auch immer so, weil das ist ein cooler Typ so ... Mit der gehe ich auch manchmal so einkaufen (männlich, 15 Jahre, kein Migrationshintergrund, Prekäre)

(7) Aber sonst, wir sprechen ja alle Deutsch als Muttersprache. Ich glaube, wenn das anders wäre, wenn wir nicht alle Deutsch als Muttersprache hätten, würde es auch noch mal anders sein, aber so spielt es für mich keine Rolle. (weiblich, 17 Jahre, Migrationshintergrund, Expeditive)

Auch wenn kaum einer der Befragten ein geschlossenes chauvinistisches Weltbild hat, sind v. a. auf Seiten der Jugendlichen ohne Migrationshintergrund stereotype Vorstellungen über Migranten in Deutschland, speziell die türkeistämmigen, weit verbreitet. Aber auch bei den Jugendlichen mit Migrationshintergrund gibt es - als Reaktion auf wahrgenommene Exklusion - Rückzugs- und Abgrenzungstendenzen.

Ob diese sich in der sensiblen Phase der bei 14- bis 17-Jährigen beginnenden verstärkten Auseinandersetzung mit gesellschaftspolitischen Themen auflösen oder zu Negativklischees und manifesten Vorurteilen verfestigen, wird das künftige gesellschaftliche Klima mit prägen - eine bleibende Herausforderung insbesondere auch für die pädagogischen Instanzen (Elternhaus, Schule, Vereine, Kirchen/Moscheegemeinden etc.).

Open Access Dieses Kapitel wird unter der Creative Commons Namensnennung - Nicht kommerziell 2.5 International Lizenz (http://creativecommons.org/licenses/by-nc/2.5/deed.de) veröffentlicht, welche für nicht kommerzielle Zwecke die Nutzung, Verbreitung und Wiedergabe in jeglichem Medium und Format erlaubt, sofern Sie den/die ursprünglichen Autor(en), den Titel des Werks und die Quelle ordnungsgemäß nennen, einen Link zur Creative Commons Lizenz beifügen und im Falle einer Abwandlung durch einen entsprechenden Hinweis deutlich erkennbar machen, dass Änderungen vorgenommen wurden.

Die in diesem Kapitel enthaltenen Bilder und sonstiges Drittmaterial unterliegen ebenfalls der genannten Creative Commons Lizenz, sofern sich aus der Abbildungslegende nichts anderes ergibt. Sofern das betreffende Material nicht unter der genannten Creative Commons Lizenz steht und die betreffende Handlung nicht nach gesetzlichen Vorschriften erlaubt ist, ist auch für die oben aufgeführten nicht-kommerziellen Weiterverwendungen des Materials die Einwilligung des jeweiligen Rechteinhabers einzuholen. 